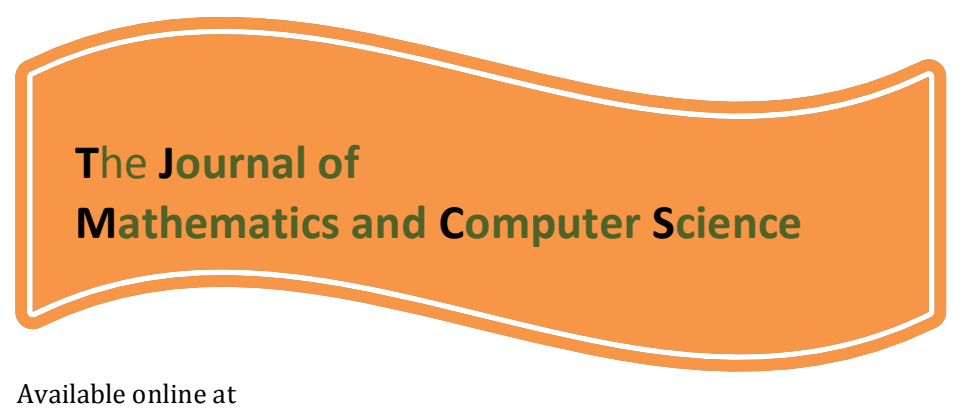

\title{
http://www.T]MCS.com
}

The Journal of Mathematics and Computer Science Vol. 4 No.1 (2012) 48 - 52

\section{Monte Carlo simulation for numerical integration based on antithetic variance reduction and Halton's sequences}

\author{
Farshid Mehrdoust \\ Department of Applied Mathematics, \\ Faculty of Mathematical Sciences, University of Guilan \\ Rasht, Iran \\ fmehrdoust@guilan.ac.ir
}

Received: December 2011, Revised: March 2012

Online Publication: May 2012

\begin{abstract}
Many applications, for instance in finance and in physics, require the calculation of high dimensional integrals. The Monte Carlo and quasi Monte Carlo methods are frequently used to approximate them. In this paper, we propose a new quasi Monte Carlo algorithm based on antithetic variance reduction and Halton's sequences for numerical integration. Efficiency of the new algorithm compared to the standard Monte Carlo algorithm is shown using example.
\end{abstract}

Key word: Monte Carlo simulation, Multidimensional integration, Antithetic ariance reduction, Halton's sequences

\section{INTRODUCTION}

The problem of evaluating integrals of high dimension is an important task since it appears in many important scientific applications of financial mathematics, economics, environmental mathematics and statistical physics. Randomized (Monte Carlo) algorithms have proven to be very efficient in solving multidimensional integrals in composite domains [1]. However, it is well known fact that the number of realization to be generated and deterministic problems to be solved often becomes very large and therefore the CPU time requirements may increase. In order to improve the efficiency of MC based on methods a variety of variance reduction techniques have been developed during the last decades [1-4, 7]. Finding ways of constructing estimators with smaller 
variance can often lead to an improvement in the efficiency as well. The efficiency is a quality measure for estimators that take into account both their variance and computation time. Generally, variance reduction technique may increase the accuracy of the estimator by a decreased sample standard deviation, instead of larger samples [2, 3]. Quasi Monte Carlo methods can be succinctly described as deterministic versions of Monte Carlo methods. Determinism enters in two ways, namely, by working with deterministic points rather than random samples and by the availability of deterministic error bounds instead of the probabilistic Monte Carlo error bounds. The connections between quasi Monte Carlo methods and uniform pseudorandom numbers arise in the theoretical analysis of various methods for the generation of uniform pseudorandom numbers. The pseudorandom sequences simulate random samples from a $\mathrm{U}(0,1)$ distribution and quasi-random sequences correspond to samples from a $\mathrm{U}(0,1)$ distribution. In [6,7] one can find several quasi-random sequences, such as Halton sequences, Sobol sequences, Faure sequences, and Niederreiter sequences.

In this paper, we present a new numerical algorithm for evaluating multidimensional integrals based on antithetic variance reduction and Halton's sequences.

\section{A. The Probable error in randomized algorithm}

Suppose that $X \sim N\left(\mu, \sigma^{2}\right)$ is a random variable. We have

$$
P\left(x^{\prime}<X<x^{\prime \prime}\right)=\frac{1}{\sigma \sqrt{2 \pi}} \int_{x^{\prime}}^{x^{\prime \prime}} \exp \left[-\frac{1}{2}\left(\frac{x-\mu}{\sigma}\right)^{2}\right] d x
$$

Let us $x-\mu=\sigma t$. Then we can write

$$
P\left(x^{\prime}<X<x^{\prime \prime}\right)=\frac{1}{\sqrt{2 \pi}} \int_{t_{1}=\frac{x^{\prime}-\mu}{\sigma}}^{t_{2}=\frac{x^{\prime \prime}-\mu}{\sigma}} \exp \left(-\frac{t^{2}}{2}\right) d t
$$

Consider the error function, $\Phi(x)=\frac{1}{\sqrt{2 \pi}} \int_{0}^{x} \exp \left(-\frac{t^{2}}{2}\right) d t$, therefore,

$$
P\left(x^{\prime}<X<x^{\prime \prime}\right)=\frac{1}{2}\left\{\Phi\left(t_{2}\right)-\Phi\left(t_{1}\right)\right\}
$$

According the rule of three sigmas [SOBOL] and the last formula it follows that

$$
P(\mu-3 \sigma<X<\mu+3 \sigma)=\Phi(3)=0.997
$$

Now, consider $x^{\prime}=\mu-r$ and $x^{\prime \prime}=\mu+r$ where $r=0.6745 \sigma$. Then

$$
P(\mu-\sigma<X<\mu+\sigma)=\Phi(0.6745)=0.5
$$

This equation show that the values of deviating from $\mu$ by more than $r$ and values deviating from $\mu$ by less than $r$ are equally probable. Therefore, $r$ is called the probable error of $X$. 


\section{EVAlUATIONG MULTIPLE INTEGRALS USING STANDARD MONTE CARLO METHODS}

The Monte Carlo methods give statistical estimates for any linear functional of the solution by performing random sampling of a certain random variable $X$, whose mathematical expectation is the desired functional $J$, i.e., $E[X]=J$. Using $N$ independent values (realizations) of $X: X_{1}, X_{2}, \ldots, X_{N}$, an approximation to $J$,

$$
J \approx \frac{1}{N}\left(X_{1}+X_{2}+\cdots+X_{N}\right)
$$

can then be computed.

Using (5) we have

$$
P\left(\left|\frac{1}{N} \sum_{j=1}^{N} X_{j}-J\right|<\frac{3 \sigma}{\sqrt{N}}\right) \approx 0.997
$$

In practical computations, it is more convenient to use the probable error $r_{N}=\frac{0.6754 \sigma}{\sqrt{N}}$.

Let us approximately compute the following multiple integral

$$
I=\iint \underset{\Omega}{\ldots} h(x) d x
$$

Consider the density function $p(x)$ on region $\Omega=[0,1]^{s}, s \geq 1$. Let random variable $X$ as

$$
\Theta=\frac{h(X)}{p(X)},
$$

where random variable $X$ with the distribution $p(x)$. Therefore, we have

$$
E[\Theta]=\iint \ldots \int \frac{h(x)}{p(x)} p(x) d x=I .
$$

Now let us consider $N$ independent identically distributed random variable $X_{1}, X_{2}, \ldots, X_{N}$, then for sufficiently large $N$

$$
I \approx \frac{1}{N} \sum_{i=1}^{N} \frac{h\left(x_{i}\right)}{p\left(x_{i}\right)} .
$$

Theorem 1. Based on the probable error, the value $\sigma^{2}=\operatorname{var}(\Theta)$ will be minimized, when the density function $p(x)$ is proportional to $|h(x)|$, i.e.

Proof. See [1]

$$
p(x)=\frac{|h(x)|}{\iint_{\Omega} \ldots|h(x)| d x}
$$

\section{The Halton Sequence}

One of the most important low-discrepancy sequences, used by many researchers, is the Halton sequence. Its definition is based on the radical inverse function defined as follows

$$
\Phi_{p}=\frac{b_{0}}{p}+\frac{b_{1}}{p^{2}}+\cdots+\frac{b_{m}}{p^{m+1}},
$$


where $p$ is a prime number and $n$ is given by $n=b_{0}+b_{1} p+\cdots+b_{m} p^{m}$, with integer $0 \leq b_{j}<p$. The Halton sequence, $X_{n}$, in s-dimensional is then defined as

$$
X_{n}=\left(\Phi_{p_{1}}, \Phi_{p_{2}}, \ldots, \Phi_{p_{s}}\right), \quad n=0,1, \ldots
$$

where the integer numbers $\mathrm{p}_{1}, \ldots, \mathrm{p}_{\mathrm{s}}$ are greater than one and pair wise prime. Most of the times, they are chosen as the first s primes. In this paper, we use optimal Halton sequence

$[6,7]$.

\section{ANTITHETIC VARIANCE REDUCTION}

This method was introduced by Hammersley and Morton in 1956. It can be applied easily to most problems and often produces at least a modest variance reduction. In its simplest form, it is based on the idea that instead of estimating $\mu$ by the average of i.i.d. random variables having expectation $\mu$, use pairs of negatively correlated random variables, again with expectation $\mu$. We expect the quantity $\frac{1}{2}[h(U)+h(1-U)]$ to have smaller than $h(U)$, where $U \sim U\left([0,1]^{s}\right)$. If $h(U)$ is too small, then $h(1-U)$ will have a good chance of being too large, and conversely. Therefore, we define the estimator

$$
\frac{1}{N} \sum_{i=1}^{N} \frac{1}{2}\left[h\left(U_{i}\right)+h\left(1-U_{i-1}\right)\right], \quad U_{i} \sim U\left([0,1]^{s}\right), i=1,2, \ldots, N
$$

\section{NUMERIAL EXPERIMENTS}

Here, we present a numerical example to show the effectiveness of our algorithm. We compare the proposed algorithm and standard antithetic Monte Carlo algorithm using relative error. In this examples, our algorithm gives good estimations with reduced standard deviation. Simulations are carried on a on Intel(R), Core(TM)2 CPU, $1.83 \mathrm{GHz}$ personal machine.

Example 1. $I=\iiint_{\Omega} \int \exp \left(x_{1}+x_{2}+x_{3}+x_{4}\right) d x_{1} d x_{2} d x_{3} d x_{4}, \Omega=[0,1]^{4}$. The referent value of the integral is approximately equal to 8.7172116201 .

Table 1. The relative error (in absolute value) and the standard deviation for different values of the simulations

\begin{tabular}{||c|c|c||}
\hline \hline $\begin{array}{c}\text { NUMBER OF } \\
\text { SIMULATION }\end{array}$ & $\begin{array}{c}\text { SANDARD ANTITHETIC VARIATES } \\
\text { (REL. ERROR, STD. DIV) }\end{array}$ & $\begin{array}{c}\text { HALTON-BASED ANTITHETIC VARIATES } \\
\text { (REL. ERROR, STD. DIV) }\end{array}$ \\
\hline $\mathrm{N}=500$ & $0.0084,1.8619$ & $0.0019,1.8276$ \\
\hline $\mathrm{N}=1000$ & $0.0038,1.8257$ & $0.0018,1.7913$ \\
\hline
\end{tabular}




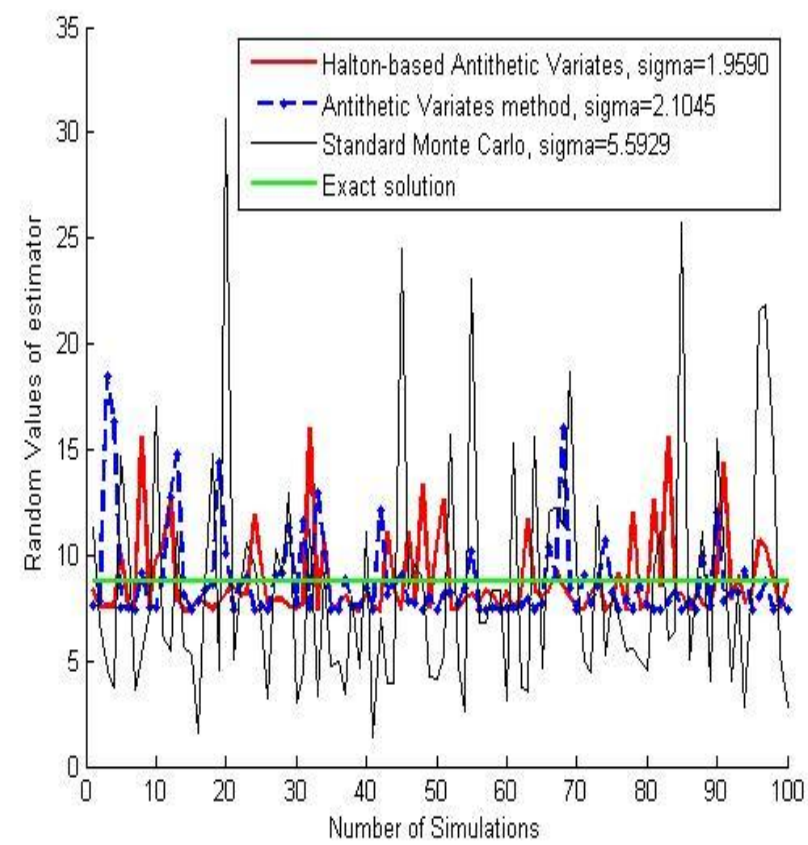

Figure 1. Comparison the standard deviations for three randomized algorithms

\section{CONCLUDING REMARKS}

Monte Carlo integration is numerical quadrature using random numbers. In other words, Monte Carlo integration methods are algorithms for the approximate evaluation of definite integrals. The regular algorithms evaluate the integrand over a grid, whereas Monte Carlo methods randomly choose the points at which the integrand is evaluated. Keep in mind that similar to other numerical techniques, the Monte Carlo evaluation of integrals is only an approximation. Although Monte Carlo is efficient for multidimensional integrals, it is slow, requiring many samples for convergence. In this paper, we proposed a simple and new method to estimate of multidimensional integrals. The proposed method is more accurate than the standard Monte Carlo and antithetic Monte Carlo methods.

\section{REFERENCES}

[1] I.T Dimov, Monte Carlo Methods for Applied Scientists.World Scientific, London, Singapore, 2008.

[2] B. Fathi Vajargah and F. Mehrdoust, Some Advantages on Monte Carlo Integration using Variance Reduction Procedures, International Journal of Advanced Research in Computer Science, 2010.

[3] J. M. Hammersly, and D. C. Handscomb.Monte Carlo methods, Landon Methuen and Company, 1964.

[4] A. Karaivanova, I. Dimov, Error analysis of an adaptiv Monte Carlo method for numerical integration, Mathematics and Coputers in Simulation, 1998, 47, 201-213.

[5] E. L. Lehmann, Some concepts of dependence. Annals of Mathematical Statistics, 1966,47,1137-1153.

[6] R. Y. Rubinstein, Simulation and the Monte Carlo method, John Wiley \& Sons, New York, 1981.

[7] I. M. Sobol, Quasi Monte Carlo methods, Progress in nuclear energy, 1990. 